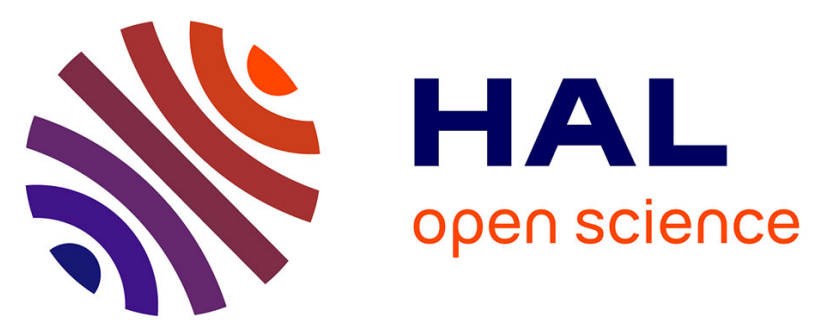

\title{
Le microbiote comme outil thérapeutique dans l'allergie alimentaire
}

Bertrand Evrard, B. Bonnet, Grégory Jubelin, Annick Bernalier-Donadille

\section{To cite this version:}

Bertrand Evrard, B. Bonnet, Grégory Jubelin, Annick Bernalier-Donadille. Le microbiote comme outil thérapeutique dans l'allergie alimentaire. Revue francaise d'allergologie, 2019, 59 (3), pp.152153. 10.1016/j.reval.2019.02.223 . hal-02154533

\section{HAL Id: hal-02154533 \\ https://hal.science/hal-02154533}

Submitted on 22 Oct 2021

HAL is a multi-disciplinary open access archive for the deposit and dissemination of scientific research documents, whether they are published or not. The documents may come from teaching and research institutions in France or abroad, or from public or private research centers.
L'archive ouverte pluridisciplinaire HAL, est destinée au dépôt et à la diffusion de documents scientifiques de niveau recherche, publiés ou non, émanant des établissements d'enseignement et de recherche français ou étrangers, des laboratoires publics ou privés.

\section{다)(1) $(5$}

Distributed under a Creative Commons Attribution - NonCommercial| 4.0 International 


\title{
Le microbiote comme outil thérapeutique dans l'allergie alimentaire
}

\author{
Microbiota as a therapeutic tool in food allergy
}

B. Evrard ${ }^{a, b, *}$, B. Bonnet ${ }^{a, b}, G$. Jubelin ${ }^{c}, A$. Bernalier-Donadille ${ }^{c}$

a UMR 1019 UNH, ECREIN, laboratoire d'immunologie, UFR médecine, université Clermont-Auvergne, 63000 Clermont-Ferrand, France

b Service d'immunologie, 3e étage centre de biologie, CHU de Clermont-Ferrand, 58, rue Montalembert, 63003 Clermont-Ferrand cedex 1, France

C UMR 454 MEDIS, Université Clermont Auvergne, INRA, 63000 Clermont-Ferrand, France

Mots Clefs : microbiote intestinal, allergie alimentaire, probiotique, tolérance, immunothérapie orale Keywords : gut microbiota, food allergy, probiotic, tolerance, oral immunotherapy

Conflit d'intérêt : aucun

Auteur correspondant :

Bertrand Evrard

CHU Gabriel-Montpied, Centre de Biologie $3^{\text {ème }}$ étage, Service d'Immunologie, 58 rue Montalembert, 63003 Clermont-Ferrand cedex 1, France 
Au cours des dernières décennies, l'épidémiologie de l'allergie alimentaire a fortement évolué - bien que les chiffres restent à prendre avec précaution - avec des augmentations simultanées et importantes de la prévalence, de la sévérité des manifestations cliniques et du risque de persistance à l'âge adulte ${ }^{1,2}$. Partant de ce constat, et de celui qu'une évolution si rapide dans le temps ne pouvait être imputée aux seuls facteurs génétiques prédisposants, il est largement admis que des facteurs environnementaux sous-tendent le développement de l'allergie. Dès les années 80 , Strachan proposait sa célèbre théorie de l'hygiène qui pourrait expliquer ces changements épidémiologiques, notamment dans les pays occidentaux. En effet, dans une lecture moderne, cette théorie suppose que des facteurs environnementaux multiples liés à notre mode de vie - dont l'excès d'hygiène corporelle et des surfaces, la large utilisation des antibiotiques, les modifications de l'alimentation, la décontamination de l'eau de boisson, l'augmentation des naissances par césarienne... - induisent durant la petite enfance une moindre exposition aux micro-organismes telluriques non pathogènes ainsi qu'une modification de nos microbiotes, particulièrement du microbiote intestinal. Or ce dernier, via la reconnaissance des bactéries commensales de la lumière digestive par les cellules dendritiques $\mathrm{CD103}$ + de la muqueuse intestinale et par la sécrétion de métabolites (comme les acides gras à courte chaîne), est indispensable à l'éducation du système immunitaire et à l'équilibre des sous-populations lymphocytaires T CD4+. L'altération du microbiote intestinal, c'est-à-dire la dysbiose, consécutive aux modifications de notre environnement induirait ainsi un défaut de maturation des mécanismes d'immunorégulation, spécialement de la génération des lymphocytes T régulateurs FoxP3+, médié au moins en partie par des phénomènes épigénétiques. Dans l'allergie, la dysbiose induit donc avant tout une rupture de tolérance, et seulement secondairement un excès de Th2 (et de Th1 dans les phases d'exacerbation). Néanmoins, il est intéressant de rajouter que l'hypothèse de l'hygiène est actuellement en partie remise en cause par une nouvelle hypothèse, celle de l'exposition duale aux allergènes, qui suggère que la sensibilisation aux aliments se produirait tôt dans la vie via l'exposition par voie cutanée à de faibles doses d'allergènes, alors que la consommation précoce de protéines alimentaires induirait plutôt une tolérance orale ${ }^{3}$.

L'implication du microbiote intestinal dans la physiopathologie de l'allergie IgE-dépendante a été progressivement confirmée à travers des études corrélant la présence des facteurs environnementaux précédemment décrits avec celle chez les patients d'une dysbiose intestinale associée à une augmentation du risque de survenue de l'allergie. Le microbiote des enfants allergiques présentent ainsi des anomalies de répartition entre les différentes espèces et une complexité bactérienne globalement plus faible que les enfants sains, mais il reste difficile d'établir une corrélation claire entre des taxons bactériens spécifiques et le développement de l'allergie ${ }^{2}$. A la naissance, le tube digestif du nouveau-né est stérile, puis rapidement les microbes de la mère et de l'environnement immédiat commencent à le coloniser. Le microbiote initial prédominant, maintenu durant toute la lactation, est composé de bifidobactéries. Par la suite, le sevrage et l'introduction d'une alimentation solide modifient profondément le microbiote du nouveau-né, chez qui s'installe alors progressivement un microbiote de type adulte, c'est-à-dire dominé par la présence de 
Firmicutes et de Bacteroidetes. Cette phase de transition, vers une communauté bactérienne variée de type adulte est une étape critique dans l'apparition de la dysbiose puis de l'allergie, d'où le concept de fenêtre d'opportunité. En effet, une partie importante du déterminisme de l'allergie semblant se jouer à cette étape précoce de la vie, il s'agit sans doute du moment le plus favorable pour tenter des approches interventionnelles ciblant le microbiote comme outil thérapeutique de l'allergie. Néanmoins, les différents types d'allergie alimentaire ne se déclenchant pas aux mêmes âges et n'ayant pas toutes le même profil en termes de persistance ou guérison, il existe sans doute différentes fenêtres d'exposition, éventuellement liés au type d'aliment, à l'âge et à l'implication de bactéries intestinales immunomodulatrices différentes ${ }^{3}$. Le meilleur moment pour tenter d'obtenir une action thérapeutique efficace sur le microbiote intestinal et sur l'allergie alimentaire n'est donc pas forcément le même pour tous les types d'allergie alimentaire, mais les données manquent encore beaucoup pour conclure.

Diverses stratégies interventionnelles innovantes peuvent dès lors être envisagées afin d'utiliser les connaissances récentes sur le microbiote intestinal pour développer de nouveaux outils thérapeutiques dans l'allergie alimentaire. Nous décrirons ainsi l'utilisation des probiotiques, domaine dans lequel la découverte des propriétés immumodulatrices de Bacteroïdes fragilis, des Bactéries Filamenteuses Segmentées (SFB) ou encore d'Akkermansia muciniphila laisse espérer l'apparition d'une nouvelle génération de bactéries plus efficaces ${ }^{4,5}$. Nous envisagerons également l'usage potentiel des prébiotiques et des symbiotiques, ainsi que les tentatives de manipulation du microbiote par des approches nutritionnelles telles que des régimes riches en fibre. Ensuite, nous discuterons des potentialités de la transplantation de microbiote fécale pour les formes les plus sévères d'allergie et de celles des immunothérapies combinées, dans lesquelles les bactéries issues du microbiote pourraient jouer le rôle de véritables adjuvants bactériens ${ }^{6,7}$.

Enfin, nous ouvrirons le débat sur la notion de microbiome, au sens large, afin de montrer que dans un futur proche il ne faudra plus seulement s'intéresser au microbiote bactérien, mais bien également en même temps au virome, au mycobiome et à l'eukaryome ${ }^{8}$. L'ère de la biologie complexe et intégrative ne fait que débuter...

\section{Bibliographie}

1. Sicherer SH, Sampson HA. Food allergy: A review and update on epidemiology, pathogenesis, diagnosis, prevention, and management. J Allergy Clin Immunol. 2018;141(1):41-58. doi:10.1016/j.jaci.2017.11.003

2. Aitoro R, Paparo L, Amoroso A, et al. Gut Microbiota as a Target for Preventive and Therapeutic Intervention against Food Allergy. Nutrients. 2017;9(7). doi:10.3390/nu9070672

3. Du Toit G, Sampson HA, Plaut M, Burks AW, Akdis CA, Lack G. Food allergy: Update on prevention and tolerance. J Allergy Clin Immunol. 2018;141(1):30-40. doi:10.1016/j.jaci.2017.11.010 
4. Zhao W, Ho H-E, Bunyavanich S. The Gut Microbiome in Food Allergy. Ann Allergy Asthma Immunol. December 2018. doi:10.1016/j.anai.2018.12.012

5. Tan-Lim CSC, Esteban-Ipac NAR. Probiotics as treatment for food allergies among pediatric patients: a meta-analysis. World Allergy Organ J. 2018;11(1):25. doi:10.1186/s40413-018-0204-5

6. Tang MLK, Ponsonby A-L, Orsini F, et al. Administration of a probiotic with peanut oral immunotherapy: A randomized trial. J Allergy Clin Immunol. 2015;135(3):737-744.e8. doi:10.1016/j.jaci.2014.11.034

7. Hsiao K-C, Ponsonby A-L, Axelrad C, Pitkin S, Tang MLK, PPOIT Study Team. Long-term clinical and immunological effects of probiotic and peanut oral immunotherapy after treatment cessation: 4-year follow-up of a randomised, double-blind, placebo-controlled trial. Lancet Child Adolesc Health. 2017;1(2):97-105. doi:10.1016/S2352-4642(17)30041-X

8. Heitlinger E, Ferreira SCM, Thierer D, Hofer H, East ML. The Intestinal Eukaryotic and Bacterial Biome of Spotted Hyenas: The Impact of Social Status and Age on Diversity and Composition. Front Cell Infect Microbiol. 2017;7. doi:10.3389/fcimb.2017.00262 